\title{
Chronic Polyneuropathy
}

National Cancer Institute

\section{Source}

National Cancer Institute. Chronic Polyneuropathy. NCI Thesaurus. Code C36071.

Polyneuropathy that is persistent or long-standing in nature. 